\title{
Harnessing tactile waves to measure skin-to-skin interactions
}

\author{
Louise P. Kirsch ${ }^{1} \cdot$ Xavier E. Job ${ }^{1} \cdot$ Malika Auvray $^{1}$ (D) $\cdot$ Vincent Hayward $^{1}$ (D)
}

Accepted: 24 September 2020 / Published online: 17 November 2020

(C) The Psychonomic Society, Inc. 2020

\begin{abstract}
Skin-to-skin touch is an essential form of tactile interaction, yet there is no known method to quantify how we touch our own skin or someone else's skin. Skin-to-skin touch is particularly challenging to measure objectively, since interposing an instrumented sheet, no matter how thin and flexible, between the interacting skins is not an option. To fill this gap, we explored a technique that takes advantage of the propagation of vibrations from the locus of touch to pick up a signal that contains information about skinto-skin tactile interactions. These "tactile waves" were measured by an accelerometer sensor placed on the touching finger. Applied pressure and speed had a direct influence on measured signal power when the target of touch was the self or another person. The measurements were insensitive to changes in the location of the sensor relative to the target. Our study suggests that this method has potential for probing behaviour during skin-to-skin tactile interactions and could be a valuable technique to study social touch, self-touch, and motor control. The method is non-invasive, easy to commission, inexpensive, and robust.
\end{abstract}

Keywords Tactile interaction $\cdot$ Skin-to-skin touch $\cdot$ Self-touch $\cdot$ Social touch

\section{Introduction}

Skin-to-skin touch has broad implications for the sense of self (Merleau-Ponty, 1962; Crucianelli et al., 2013), body representation (Schütz-Bosbach \& Haggard, 2009; van Stralen et al., 2014), affective touch (McGlone et al., 2014; Cascio et al., 2019) and motor control (Blakemore et al., 2000; Bays $\&$ Wolpert, 2008). It is thus connected to intriguing problems across the domains of philosophy, psychology, and neuroscience. However, to date, no empirical method is capable of measuring how we touch the skin of a living person. Even a seemingly straightforward parameter such as the pressure applied during skin-to-skin touch is outside the reach of objective measurement.

When touching surfaces other than the skin, the net force applied can be directly measured by instrumenting the touched surfaces with load sensors interposed between the surface and a mechanical reference. For example, in grasping studies, hand-held objects are typically instrumented with load

Malika Auvray and Vincent Hayward share senior authorship

Louise P. Kirsch

kirsch@isir.upmc.fr

1 Institut des Systèmes Intelligents et de Robotique, Sorbonne Université, 4 place Jussieu, 75005 Paris, France cells connecting grip surfaces to the object (e.g. Johansson \& Westling, 1984). Such arrangements project the total interaction of the finger onto the tangential and normal directions of the touched surface. Motor behaviour can be inferred from this decomposition. Extensions of this technique using broadband sensors revealed the complexity of the fingers' mechanical interactions with surfaces (Wiertlewski et al., 2011; Klöcker et al., 2013; Gueorguiev et al., 2016).

When the touched surface is skin, it is not possible to measure the interaction by interposing an instrumented membrane between the skins, since the properties of the skin contribute to the interaction (Morrison, Löken, and Olausson, 2010; Adams et al., 2013). As far as motor behaviour is concerned, electromyography (EMG) and acoustic myography (AMG) are invaluable techniques for investigating muscle activation (Goldenberg et al., 1991; Hodges, 2019). These techniques, however, cannot provide a precise measure of the activity of an individual at the level of the fingers, even in highly constrained conditions and with sophisticated analysis techniques (Waris et al., 2018).

Here, a novel technique is introduced which is sensitive to the effects of skin-to-skin touch and which provides a signal containing information about the behaviour of the 'toucher' and the nature of the interaction. It is adapted from previous work highlighting the propagation of mechanical energy in soft tissues far from a region of contact. The effect of digital tactile interactions can be measured in the fingertip (Bensmaia and Hollins, 2003; Tanaka et al., 2011), the finger (Gu et al., 
2019; Shi et al., 2020; Tanaka et al., 2012; Manfredi et al., 2012), the whole hand (Shao et al., 2016, 2020), at least as far as in the forearm (Delhaye et al., 2012; Laput, Xiao, \& Harrison, 2016). These long-range effects are likely to result from the propagation of elastic S-waves (Vexler et al., 1999) and surface Rayleigh waves (Kirkpatrick et al., 2004) in soft tissues, with a relatively low rate of attenuation over distance. These studies have used a combination of techniques, including accelerometers and optical vibrometers. So far, tactile interactions with objects and rigid surfaces have been well documented. However, no study to date has investigated a practical way in which to measure skin-to-skin tactile interactions.

It is known that almost all mechanical sliding contacts undergo fluctuations for any speed (Akay, 2002). The fingers are no exception. When they slide on almost any surface, including skin, contact fluctuations arise from phenomena that take place at multiple length and time scales. These phenomena vary in relative importance in accordance with the material properties of the solids in contact and the relative topographies (roughness, corrugation, conformability) at molecular, mesoscopic, and macroscopic scales (Baumberger and Caroli, 2006). The friction associated with skin-to-skin touch is the result of the skin's complex material properties and intricate topography at all length scales. In fact, the sounds produced by the sliding of glabrous skin against glabrous skin (the ridged skin corresponding to the prehensile regions of the hand) are sufficiently strong to be heard and to modify perceptual behaviour (Jousmäki and Hari, 1998). These fluctuations are usually called frictional noise. For the present purpose they represent frictional signal. The role of frictioninduced fluctuations has been well documented in the rodent vibrissal system (Hipp et al., 2006; Ritt et al., 2008; Schwarz, 2016).

The intensity and spectral properties of the frictional fluctuations of skin sliding against skin depend upon numerous factors, including the gross shape of the regions in contact, the type of skin, the relative states of hydration, and the presence of lubricants or solid contaminants. Our study aimed to investigate how these fluctuations were linked to how we touch skin, including pressure applied and speed. To this end, a consumer-grade accelerometer chip was attached to a single location of the touching finger to measure cutaneous vibrations remotely from the region of contact, see Fig. 1. The captured signal was compared across conditions that varied the participants' instructed movements.

In Experiments 1 and 2, participants were instructed to vary the pressure applied during touch (gentle or firm) or their sliding speed (fast, medium, or slow), respectively. If the signal was sensitive to these behavioural features of skin-to-skin touch, then differences in the signal should be observed between these conditions (e.g. higher signal power for firm and fast compared to gentle and slow touch, respectively). In Experiment 3, the target orientation was varied such that the dorsal or ventral surface of the touched finger (i.e. the target) was facing the participant, inverting the relationship of the touching fingers with the dorsal or ventral surfaces of the target. If sensor placement was critical, then the signal should depend on target orientation.

Skin-to-skin touch can be broadly divided into actions that serve to touch one's own skin or another person's skin, with key differences between these two types of touch (Verrillo et al., 2003; Ackerley et al., 2012). It is possible that the signal obtained during skin-to-skin touch depended on the target of the touch (e.g., Schütz-Bosbach et al., 2009). In all three experiments, the target was varied to be either the participant's own skin or another person's skin, in order to ascertain that the method could be applied to both types of touch.

\section{Experiment 1}

The first experiment investigated whether the friction-induced vibration signal was sensitive to differences in the pressure applied by the toucher during skin-to-skin tactile interaction. Pairs of participants touched either their own or someone else's index finger, gently or firmly.

\section{Methods}

Participants Eighteen healthy right-handed participants were recruited (ten female, mean age: 22.8 years, $\mathrm{SD}=3.4$ ). Participants were invited to take part in the experiment in dyads but did not know each other. Half of the dyads were gender-matched. In this and in all the experiments reported here, participants were naive to the purpose of the experiment. Participants provided informed consent in accordance with the ethical standards outlined by the Declaration of Helsinki (1991). All experiments received approval from the university's ethical committee. Each experiment took approximately 30 minutes to complete, and the participants received payment for their participation.

Material The sensor, shown in Fig. 1, was a surface-mounted MEMS accelerometer (Analog Devices, model ADXL320, equiv. ADXL325, $\pm 5 \mathrm{G}$ ) on a $12 \times 12 \mathrm{~mm}$ printed circuit board, which also bore decoupling and bandwidth-setting (to $500 \mathrm{~Hz}$ ) surface-mounted capacitors. The assembly was encapsulated in epoxy resin for protection against humidity. The total mass of the sensor was $0.5 \mathrm{~g}$. The $\mathrm{X}$ and $\mathrm{Y}$ outputs were tied together to provide a single signal. The sensor was fixed on the finger such that one of the two axes aligned with the medial axis of the finger. This strategy was justified by the propagation of surface waves in human fingers that guarantees the presence of an axial component (Andrews et al., 2020). The signal was amplified by $20 \mathrm{~dB}$ with a custom-made preamplifier to enhance the signal-to-noise ratio and was 
a

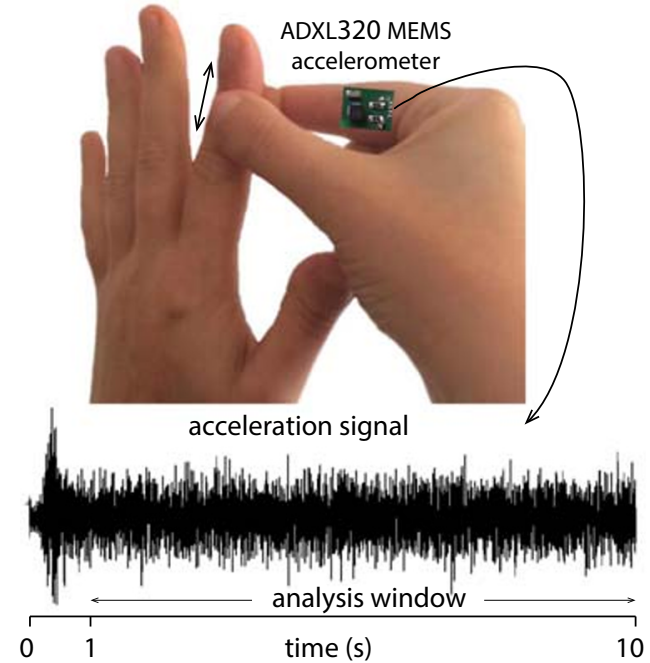

b

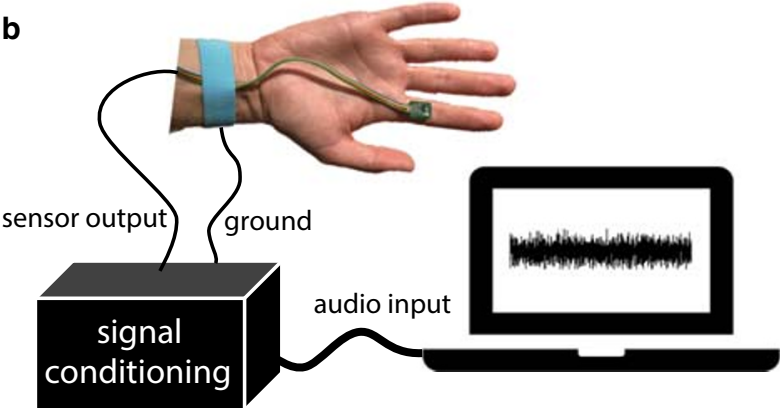

Fig. 1 Capture of tactile waves. Signals propagated from the fingertip during tactile interaction were picked up by consumer-grade accelerometer placed on the proximal phalange of the right index finger. a Schematic representation of the action performed in Experiments 1 and 3 and an example of the signal obtained showing the analysis window. b Schematic representation of the experimental set-up. The signal was acquired using a USB audio converter after $20 \mathrm{~dB}$ amplification ('signal conditioning')

acquired using a high-resolution USB audio converter (Griffin iMic USB Audio Interface). Recordings were stored as standard 16-bit audio files post-processed in MATLAB to be band-passed in the range of 35 to $300 \mathrm{~Hz}$. Mains hum at $50 \mathrm{~Hz}$ was suppressed by grounding participants to the analog neutral (RS PRO Grounding Wrist Strap \& Cord).

Procedure Participants were seated opposite each other on either side of a table approximately one metre apart. Using micropore tape, the experimenter fixed the accelerometer ventrally to the proximal phalanx of the right index finger of one of the two participants. The 'toucher' was then instructed to stroke her or his own left index finger ('self' condition) or the finger of the other participant ('other' condition). They used a precision grip posture such that the right index finger always touched the ventral glabrous region of the left index finger held upright, as illustrated in Fig. 2a. Participants always started the stroke from the fingertip of the target finger. One stroke consisted of one back and forth movement from the fingertip to the proximal phalanx and back.

Before starting the experiment, participants completed randomised practice trials of each condition. They tried to maintain a constant pace of about one stroke per second by following a metronome (sixty beats per minutes). During the experiment, a brief sound signal $(80 \mathrm{~Hz})$ cued the participants to start stroking until the signal was heard again after ten seconds. Before each trial, participants were told which target to touch, their own or the other participant's index finger, and how much to press, gently or firmly. They were free to determine what for them was gentle or firm. Each condition was randomly repeated ten times for a total of forty trials. The randomisation of the order of the touches, in all three experiments, was intended to prevent an order effect from influencing the results. Between each block, participants changed places, and the accelerometer was fixed to the other participant's index finger.

Data analysis Only the high-frequency content of the acceleration signal was considered for analysis, since the low-frequency content arises from whole limb movements and changes in orientation with respect to gravity (Morris, 1973), thus mostly holding kinematic information. The first second of each trial was excluded from the analyses to eliminate the effect of the burst of signal at the transition from a static contact to a sliding contact (see Fig. 1). To

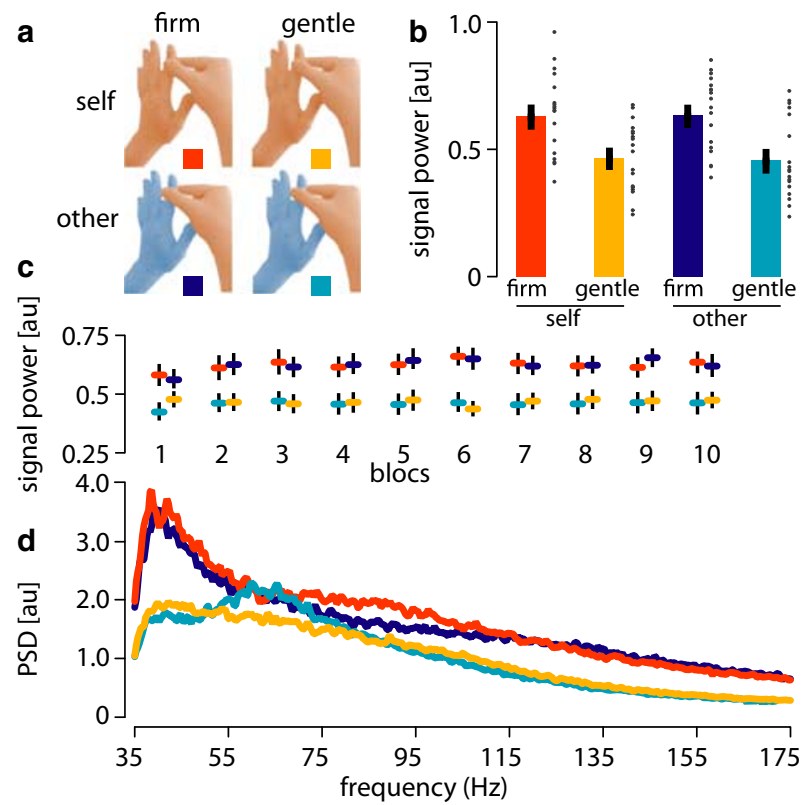

Fig. 2 Experiment 1. a Experimental design: The applied pressure could be 'firm' or 'gentle', target could be 'self' or 'other'; resulting in four conditions (colour-coded). b Total signal power of frictional fluctuations per target and pressure conditions. Black dots show individual results. Error bars show standard error of the mean (SEM). $\mathbf{c}$ Evolution of the average signal power by block number. d Averaged power spectral density (PSD) over all trials and participants for each condition 
minimise transducer noise, the signal was band-passfiltered in the range of $35-300 \mathrm{~Hz}$, which is within the textural information frequency range (Wiertlewski et al., 2010). A discrete time estimate of the average signal power was computed for each condition by assuming that the signal window was sufficiently long, a condition largely fulfilled by the audio rate sampling of $44.1 \mathrm{kHz}$. The estimates were calculated according to

$\widehat{P}_{\text {cond }}=\frac{1}{M} \sum_{i=1}^{M}\left[\frac{1}{N} \sum_{k=1}^{N}\left|a_{k}\right|^{2}\right]_{i}$

where $M$ is the number of trials per condition and $N$ the number of samples in the analysis window. A repeatedmeasures ANOVA on these averages was conducted to compare the four conditions. In addition to the analysis of signal power across the $35-300 \mathrm{~Hz}$ range, the power spectral density of the signal was estimated using Welch's method to probe differences in the spectral content profiles across conditions. The analysis was applied to the averaged power spectral density of the signal in $20 \mathrm{~Hz}$ bands (35-55, $55-75,75-95,95-115,115-135,135-155$, and 155-175 $\mathrm{Hz}$ ). Any significant interaction was followed by a post hoc $t$-test. All tests were Bonferroni-corrected for multiple comparisons.

\section{Results}

A main effect of applied pressure was observed $(F(1,17)=$ $32.70, p<0.001, \eta_{p}^{2}=0.658$ ), with higher signal power obtained when the touch was firm rather than gentle, see Fig. $2 \mathrm{~b}$. Thus, the measure was sensitive to differences in applied pressure. No effect of the target or interaction with the target was found $\left(F(1,17)=0.009, p=0.924, \eta_{p}^{2}=0.001, F(1,17)=\right.$ $0.150, p=0.703, \eta_{p}^{2}=0.009$, respectively). It should be noted that this difference was stable over time, as shown in Fig. 2c.

The difference between a gentle touch and a firm touch could also be clearly observed by inspection of the averaged power spectra over all trials and participants, see Fig. 2d, while a difference in the target was not. An analysis by $20 \mathrm{~Hz}$ frequency bands revealed a significant effect of applied pressure $\left(F(1,17)=22.616, p<0.001, \eta_{p}^{2}=0.571\right)$ and bands $\left(F(1.593,27.086)=40.391, p<0.001, \eta_{p}^{2}=0.704\right)$, and a borderline interaction between bands and pressure $\left(F(1.537,26.134)=2.942, p=0.082, \eta_{p}^{2}=0.148\right)$, but no main effect of target $(F(1,17)=0.044, \quad p=.836$, $\left.\eta_{p}^{2}=0.003\right)$, or any interaction with target. Follow-up tests showed a significant effect of pressure for all bands above $95 \mathrm{~Hz}$ (all $p<0.001$ ), as well as effects for the $35-55 \mathrm{~Hz}$ and $75-95 \mathrm{~Hz}$ bands (respectively $F(1,17)=9.301, p=$ $0.007, \quad \eta_{p}^{2}=0.354$ and $F(1,17)=8.966, \quad p=0.008$, $\left.\eta_{p}^{2}=0.345\right)$, but there was no significant difference in the $55-75 \mathrm{~Hz}$ band $\left.F(1,17)=1.130, p=0.303, \eta_{p}^{2}=0.062\right)$.

\section{Experiment 2}

The second experiment was designed to determine whether skin-to-skin, friction-induced vibrations were sensitive to differences in the magnitude of the sliding speed. Because the forearm is smoother, more uniform, and also longer than the finger, it offers a better option for the control of movement speed. Thus a simple stroking action of the forearm was used instead of the grasping action used in Experiments 1 and 3. In order to better control the speed at which participants touched the skin surface, as well as the distance travelled, participants were instructed to stroke between two fixed points of equal length marked on the ventral surface of the forearm.

\section{Methods}

Participants A new group of eighteen healthy right-handed participants completed this experiment (ten female, mean age: 23.21 years, $\mathrm{SD}=2.55$ ). Half of the dyads were gendermatched, and gender was balanced when unmatched: half of the participants were tested by a female experimenter and the other half by a male experimenter.

Procedure Participants were seated to the right of the experimenter, who placed the accelerometer on the participant's right index finger, as in Experiment 1. With a pen, the experimenter marked three spots on the ventral region of the participant's left forearm, each separated by nine centimetres (creating two sites of stimulation, sites 1 and site 2; see Fig. 3a). These marks were identical to those made beforehand on the experimenter's right forearm. Participants stroked with their right index finger the skin of their own forearm or that of the experimenter; alternating between site 1 and site 2 , to avoid habituation. It should be noted that no skin difference was expected between sites 1 and 2, so data from these two sites were averaged in the analysis. Moreover, the forearm to be touched was always positioned on the same spot, to avoid any possible configuration difference between self- and other-touch. One stroke consisted of one back and forth movement between two marks. The participants synchronised their movements to a metronome set to induce three different velocities. With a $0.33 \mathrm{~Hz}$ beat, the average speed was low, $3.0 \mathrm{~cm} / \mathrm{s}$. At $1.0 \mathrm{~Hz}$ the average speed was medium, $9.0 \mathrm{~cm} / \mathrm{s}$. At $2.0 \mathrm{~Hz}$, the average speed was fast, $18.0 \mathrm{~cm} / \mathrm{s}$. Each trial lasted nine seconds and each condition was repeated ten times in a randomised order, for a total of sixty trials. Participants 
practised each condition for a total of six trials before data were recorded.

\section{Results}

Overall, a main effect of speed was observed $(F(1.457$, $\left.24.768)=6.350, p=0.011, \eta_{p}^{2}=0.272\right)$, with more signal power at the highest speed, and a main effect of target $(F(1$, $\left.18)=12.489, p=0.003, \eta_{p}^{2}=0.424\right)$, with more signal power when touching another person than the self, but no significant interaction $\left(F(1.352,22.980)=2.910, p=0.091, \eta_{p}^{2}=0.146\right.$; Fig. 3b). Differences were found between slow and medium speeds and between medium and fast speeds $(t(18)=3.042$, $p=0.007 ; t(18)=-4.772, p<0.001$, respectively). The effect of target obtained here was likely due to an experimenter bias, since additional analysis revealed an interaction between experimenter and target difference $(F(1,16)=11.757, p=0.003$, $\left.\eta_{p}^{2}=0.424\right)$ as well as a marginal main effect of target $(F(1$, $16)=3.583, p=0.077, \eta_{p}^{2}=0.183$ ), with higher power associated with one of the two experimenters (see Supplementary Fig. S1).

A frequency band analysis indicated a main effect of bands $\left(F(1.846,31.389)=12.606, p<0.001, \eta_{p}^{2}=0.426\right)$, a main effect of target $\left(F(1,17)=9.202, p=0.007, \eta_{p}^{2}=0.351\right)$, a main effect of speed $(F(1.407,23.913)=5.566, p=0.018$, $\left.\eta_{p}^{2}=0.247\right)$, an interaction between target and bands $\left(F(2.082,35.394)=6.176, p=0.005, \eta_{p}^{2}=0.266\right)$, and an interaction between speed and bands $(F(2.411,40.992)=4.232$,

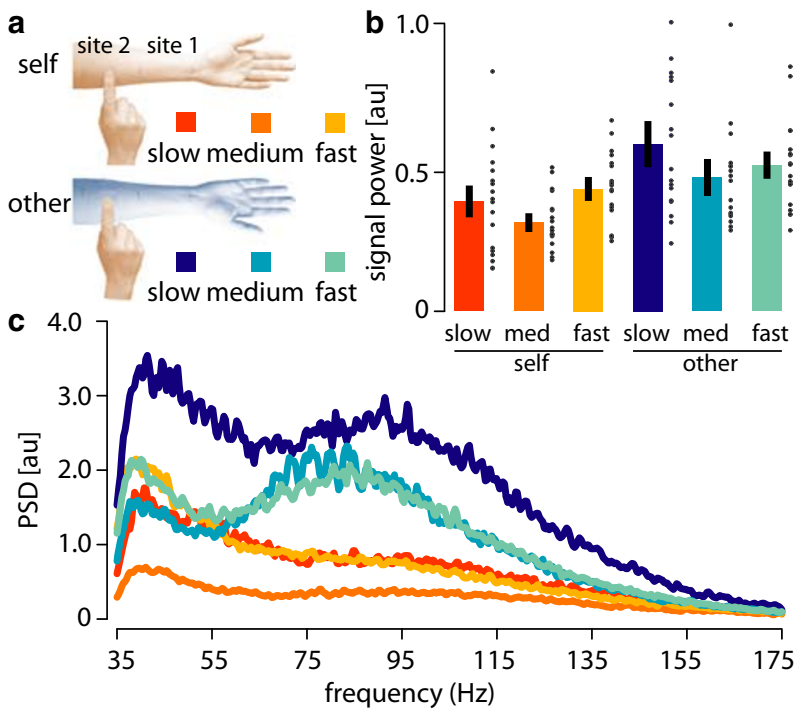

Fig. 3 Experiment 2. a Experimental design: Touching was performed at three different speeds ('slow', 'medium', 'fast') in random order between sites 1 and 2. The target could be either 'self' or 'other'; resulting in six conditions (colour-coded). b Total signal power of frictional fluctuations per target and speed condition. Black dots show individual results. Error bars show standard error of the mean (SEM). c Averaged power spectral density (PSD) across all trials and participants for all conditions $\left.p=0.016, \eta_{p}^{2}=0.199\right)$, but no interaction between target and speed and no three-way interaction with bands $(F(1.972$, $33.521)=1.690, p=0.200, \eta_{p}{ }^{2}=0.090$, see Fig. 3c). The self-other difference was seen in the bands between $55 \mathrm{~Hz}$ and $155 \mathrm{~Hz}(55-75 \mathrm{~Hz}: F(1,17)=11.197, p=0.004$, $\eta_{p}^{2}=0.397 ; \quad 75-95 \quad \mathrm{~Hz}: \quad F(1,17)=11.993, \quad p=0.003$, $\eta_{p}^{2}=0.414 ; 95-115 \mathrm{~Hz}: \quad F(1,17)=10.635, \quad p=0.005$, $\left.\eta_{p}^{2}=0.385\right)$. However, the effect of speed and the interaction between speed and target did not survive Bonferroni correction $(\alpha=0.0074)$ in any of the bands.

\section{Experiment 3}

Experiment 3 investigated whether the signal varied with the orientation of the target hand, since the location of the sensor relative to target may have influenced the signal.

\section{Methods}

Participants A new group of eighteen right-handed participants completed the experiment (nine female, mean age: 23.6 years, $\mathrm{SD}=3.6$ ). Participants were invited to take part in the experiment in dyads, but they did not know each other. As in Experiments 1 and 2, gender was balanced across dyads.

Procedure As in Experiment 1, participants were seated opposite each other on either side of a table approximately one metre apart. The accelerometer was placed on the right index finger of one participant of the dyad, who would be the participant performing the touch. The accelerometer was fixed in the same position as in Experiments 1 and 2; thus the distance between the sensor and the regions of contact varied with target orientation. Participants performed the same action as in Experiment 1 (precision grip), with the sole difference being the orientation of the touched index finger (i.e. target orientation; see Fig. 4a). In the 'outwards' condition, the palm of the target hand faced away from the toucher (i.e. the active index of the toucher was in contact with the glabrous skin on the ventral side of the target finger, and the thumb with the hairy skin on the dorsal side). In the 'inwards' condition, the palm of the target hand faced towards the toucher (i.e. the reversed configuration). As in Experiments 1 and 2, the 'toucher' was instructed to stroke their own left index finger ('self' condition) or the finger of the other participant ('other' condition). No instruction was given concerning the pressure to be applied. Participants were encouraged to keep a constant speed by the same method as in Experiment 1. Each condition was repeated ten times for a total of forty randomised trials. After those trials, the two participants changed places and the accelerometer was attached to the new toucher. 


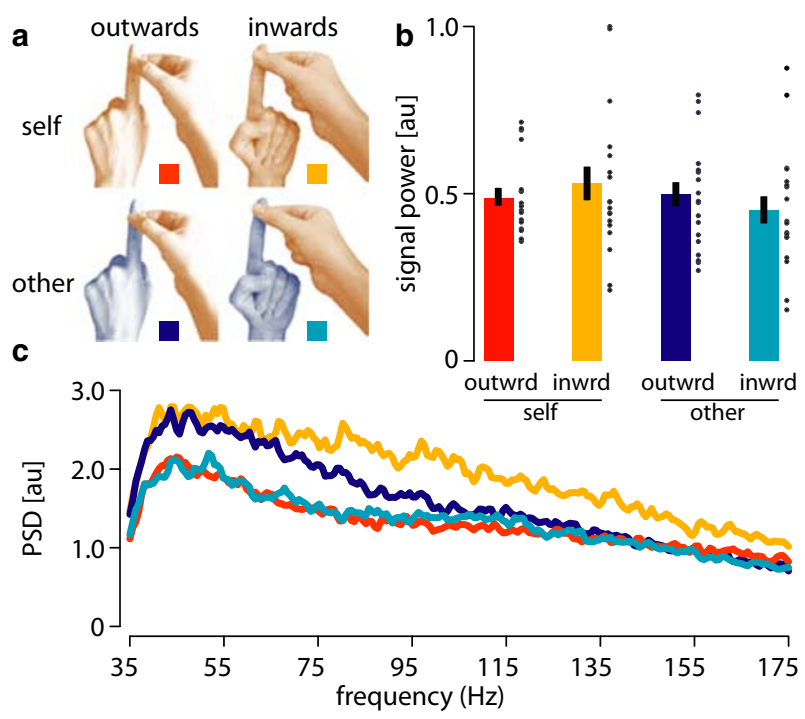

Fig. 4 Experiment 3. a Experimental design: Target orientation could be 'outwards' or 'inwards', target could be either 'self' or 'other'; resulting in four conditions (colour-coded). b Total signal power of frictional fluctuations per target and target orientation. Black dots show individual results. Error bars show standard error of the mean (SEM). c Averaged power spectral density across all trials and participants for each condition

\section{Results}

The results showed no effect of target or target orientation (target: $F(1,17)=1.724, p=0.207, \eta_{p}^{2}=0.092$; target orientation: $\left.F(1,17)=0.002, p=0.969, \eta_{p}^{2}=0.000\right)$, but showed an interaction between target and skin type $(F(1,17)=6.393$, $\left.p=0.022, \eta_{p}^{2}=0.273\right)$, see Fig. $4 \mathrm{~b}$. However, none of the post hoc $t$ tests survived Bonferroni correction, suggesting no significant impact of the orientation of the target.

An analysis by frequency bands, Fig. $4 \mathrm{c}$, revealed a main effect of bands $(F(1.513,25.725)=27.472, \quad p<0.001$, $\left.\eta_{p}^{2}=0.618\right)$, an interaction between target and skin type $\left(F(1,17)=5.239, p=0.035, \eta_{p}^{2}=0.236\right)$, and a three-way interaction with bands $(F(1.815,30.860)=5.221, p=0.013$, $\left.\eta_{p}^{2}=0.235\right)$. Follow-up analyses yielded no significant results (no main effect of target, skin, or interaction survived the Bonferroni correction).

\section{Discussion}

Skin-to-skin touch is challenging to measure objectively, yet it presents a number of intriguing problems that span neuroscience, psychology, and philosophy. Here, we tested the efficacy of a measure of skin-to-skin tactile behaviour that took advantage of the frictional fluctuations propagating in soft tissues (Shao et al., 2016, 2020). Frictional fluctuations in soft tissues such as the skin have been successfully measured during tactile interactions with objects and rigid surfaces $(\mathrm{Gu}$ et al., 2019; Manfredi et al., 2012; Shao et al., 2016, 2020; Tanaka et al., 2011); however, such methods have not been applied to skin-to-skin touch. Participants were instructed to stroke skin surfaces while an accelerometer was fixed to their touching finger. The recorded signal contained information about the vibrations elicited during touch. Participants varied the strength of their touch, their movement speed, and the orientation of the target, as well as the target identity (selftouch vs touching another's skin).

The analysis relied on the total signal power and the distribution of this power in specific frequency bands. The signal exhibited considerable variability between individuals; however, this limitation is shared by most other physiological signal measurements including pupil dilation (e.g. Einhäuser et al., 2008; Wierda et al., 2012), skin conductance (e.g. Tronstad et al., 2010; van Dooren et al., 2012), electromyography (e.g. Goldenberg et al., 1991), respiration (e.g. Boiten et al., 1994; Valderas et al., 2015), and heart rate (e.g. Appelhans and Luecken, 2006; Garfinkel et al., 2015). Despite high inter-individual variability, useful information was extracted from the signal, allowing comparisons across experimental conditions.

Experiment 1 showed a clear effect of touch strength when participants were instructed to apply either gentle or firm pressure. The signal power was significantly higher during firm compared to gentle touch. This demonstrates that a consumergrade accelerometer is able to capture tactile signals and can be used as a proxy of the force applied during skin-to-skin touch. Therefore, the method is able to detect differences in the pressure applied during skin-to-skin touch.

Experiment 2 showed that the signal was sensitive to the speed with which participants touched the skin. The relationship between sliding speed and signal power, however, was complex. The medium speed $(9 \mathrm{~cm} / \mathrm{s})$ elicited significantly lower signal power than the faster speed $(18 \mathrm{~cm} / \mathrm{s})$ and the slower speed $(3 \mathrm{~cm} / \mathrm{s})$. There may be several reasons why the relationship between movement speed and signal power was not monotonic. Participants probably moved less smoothly at slower speeds (Guigon et al., 2019). Jerky movements may have caused bursts of signal at the slowest speed. The observation of greater signal power at the highest speed (18 $\mathrm{cm} / \mathrm{s}$ ) is in line with our initial hypothesis, since greater frictional energy was dissipated during the same time window. However, future research should investigate a wider range of movement speeds.

The positioning of a single sensor relative to the source of contact may have had an effect on the signal obtained, particularly with differences across experimental conditions. Experiment 3 assessed the influence of target orientation on the signal obtained during skin-to-skin touch. In Experiment 1 , participants gripped the finger when it was oriented with the 
dorsal surface facing them. In Experiment 3, the target orientation was manipulated to be either the same, as in Experiments 1, or oriented with the ventral surface facing the toucher. The signal power did not vary systematically with target orientation, suggesting that a similar signal would have been obtained from a sensor placed on the active thumb rather than active index finger. In practice, this means that experimenters are not constrained by specific placements of the sensor on the active hand.

Several lines of evidence suggest that we may touch ourselves differently from others; this is the case, for example, in the 'touchant-touché' phenomenon (Merleau-Ponty, 1962; Husserl, 1989; Schütz-Bosbach et al., 2009). The literature also suggests that self-touch is perceived to be less intense than touch from another person or surface (Blakemore et al., 2000; Shergill et al., 2003; Bays \& Wolpert, 2008; Kilteni \& Ehrsson, 2017). This effect is attributed to motor control mechanisms that predict the sensory consequences of one's own actions using information from the motor command (i.e. efference copy). Self-generated somatosensory signals are therefore thought to be perceptually cancelled or reduced relative to externally generated somatosensory signals. Thus, to compensate for the perceived differences in the intensity of touch, participants may exert more pressure when touching themselves than when touching another person. In Experiments 1 and 3, participants touched themselves or another person in dyads. The target had no influence on signal power. In Experiment 2, one of two experimenters was the 'other' target. Stronger signal power was found when participants touched another person. Further analyses revealed that the signal was higher with one of the two experimenters. Overall, our results did not show clear differences between touching one's own skin and another person's skin. This finding may seem surprising given the known differences between touch applied to one's own compared to another person's skin (Verrillo et al., 2003; Ackerley et al., 2012). However, the lack of difference may reveal the existence of a robust motor invariant that is insensitive to the target of touch, particularly under the conditions of Experiment 1. Several motor invariants related to net force applied by the fingers have been documented (Feldman, 1980; Latash et al., 2007). In Experiment 2, having only two 'other' targets may have reduced variability and introduced additional factors such as skin hydration and also possible gender effects (that were balanced in Experiment 1 and 3, as shown in Supplementary Fig. S1). This result suggests that our method could be applied to differentiate between targets. Future studies could investigate this result further and use this method to assess whether we touch ourselves differently after touching someone else, and vice versa. Moreover, one way to quantify the subjective force that participants apply to themselves versus another person could be to use a force sensor and ask them to estimate how much pressure they used on themselves versus the other person.
Whether subjective estimates of force map directly to their behaviour when actually interacting with skin surfaces is an intriguing question for future research. These future studies could also investigate the relative advantages of different stroking actions to extract specific types of information from the vibration signal.

The experiments reported here build upon previous research that investigated frictional fluctuations across the skin during tactile interactions with objects and rigid surfaces $(\mathrm{Gu}$ et al., 2019; Manfredi et al., 2012; Shao et al., 2016, 2020; Tanaka et al., 2011). Previous studies have shown that information about objects (Shao et al., 2016, 2020) and rigid surfaces (Gu et al., 2019; Tanaka et al., 2011) that are touched can be extracted from signals measured on the skin that reflect surface waves. Here, we show that this method can also be applied to skin-to-skin touch, which provides further avenues of promising research in the behavioural sciences.

Our results were obtained using spectral density analyses, including total signal power and power spectral density in broad frequency bands. However, in natural touch, cutaneous vibrations are almost always non-stationary signals, which means that the generating processes vary over time. In our study, power spectral density analyses were adequate for the investigated factors because the participants were instructed to repeat the same action over relatively long periods of time. Future research based on the analysis of time-varying phenomena could certainly be possible, for example, with shorttime Fourier analysis.

Future research may also be aimed at estimating the source of touch, the type of action executed, or even the type of skin touched, from vibration signals measured in the hands. For example, studies aimed at differentiating between hairy and glabrous skin, which is additionally relevant to the nascent field of affective touch, or which body site has been touched would be a fruitful line of research. Blind source separation analysis techniques (Comon and Jutten, 2010) could be used, since the frictional fluctuations come from sources arising from phenomena associated with different length scales. Another direction would be to increase the number of accelerometer sensors across the hand as in Shao et al. (2016, 2020), who used up to forty-two sensors. Finally, an abundance of tools based on machine learning techniques are now available that are able to extract information from complex signals. Such methods could be used to decode behavioural interactions from the resulting tactile vibrations.

To conclude, the results of this study demonstrated the direct measurement of cutaneous vibrations resulting from friction elicited by skin-to-skin contact. We showed that the signal is primarily sensitive to the pressure applied and the speed of tactile interactions. The use of one or several accelerometers, in combination with other neuroscientific tools such as functional magnetic resonance imaging (fMRI), magnetoencephalography (MEG), or electroencephalography (EEG) (e. g. Mannini et al., 2013), has significant potential for probing 
behaviour during skin-to-skin tactile interactions, opening avenues for future research investigating a variety of factors underlying self-touch as well as social touch and motor control.

Supplementary Information The online version contains supplementary material available at https://doi.org/10.3758/s13428-020-01492-3.

Acknowledgements We thank Agnès Roby-Bramy and Yon Visell for their insightful discussions. This work was supported by Agence nationale de la recherche grant ANR-16-CE28-0015 "Developmental Tool Mastery" led by Alessandro Farné. The authors declare that no competing interests exist.

Open practices statement The data for all experiments as well as the script to filter and analyse the data are available on the OSF repository and can be accessed via this link: https://osf.io/7gw5z/?view_only= 7d351d4a7b6a443392157da6bb643a90

\section{References}

Ackerley, R., Olausson, H., Wessberg, J., \& McGlone, F. (2012). Wetness perception across body sites. Neuroscience Letters, 522(1), 73-77.

Adams, M. J., Johnson, S. A., Lefèvre, P., Lévesque, V., Hayward, V., André, T., \& Thonnard, J.-L. (2013). Finger pad friction and its role in grip and touch. Journal of the Royal Society Interface, 10(80), 20120467.

Akay, A. (2002). Acoustics of friction. The Journal of the Acoustical Society of America, 111(4), 1525-1548.

Andrews, J. W., Adams, M. J., \& Montenegro-Johnson, T. D. (2020). A universal scaling law of Mammalian touch. Science Advances, In press.

Appelhans, B. M., \& Luecken, L. J. (2006). Heart rate variability as an index of regulated emotional responding. Review of General Psychology, 10(3), 229-240.

Baumberger, T., \& Caroli, C. (2006). Solid friction from stick-slip down to pinning and aging. Advances in Physics, 55(3-4), 279-348.

Bays, P. M., \& Wolpert, D. M. (2008). Predictive attenuation in the perception of touch. In P. Haggard, Y. Rosetti, \& M. Kawato (Eds.), Sensorimotor foundations of higher cognition (Vol. 22, pp. 339-358). Oxford University Press.

Bensmaia, S. J., \& Hollins, M. (2003). The vibrations of texture. Somatosensory \& Motor Research, 20(1), 33-43.

Blakemore, S.-J., Wolpert, D. M., \& Frith, C. D. (2000). Why can't you tickle yourself? Neuroreport, 11(11), R11-R16.

Boiten, F. A., Frijda, N. H., \& Wientjes, C. J. E. (1994). Emotions and respiratory patterns: review and critical analysis. International Journal of Psychophysiology, 17(2), 103-128.

Cascio, C. J., Moore, D., \& McGlone, F. (2019). Social touch and human development. Developmental Cognitive Neuroscience, 35, 5-11.

Comon, P., \& Jutten, C. (2010). Handbook of blind source separation: independent component analysis and applications. Academic Press.

Crucianelli, L., Metcalf, N. K., Fotopoulou, A. K., \& Jenkinson, P. M. (2013). Bodily pleasure matters: velocity of touch modulates body ownership during the rubber hand illusion. Frontiers in Psychology, 4, 703 .

Delhaye, B., Hayward, V., Lefèvre, P., \& Thonnard, J.-L. (2012). Texture-induced vibrations in the forearm during tactile exploration. Frontiers in Behavioral Neuroscience, 6(37), 1-10.

Einhäuser, W., Stout, J., Koch, C., \& Carter, O. (2008). Pupil dilation reflects perceptual selection and predicts subsequent stability in perceptual rivalry. Proceedings of the National Academy of Sciences, 105(5), 1704-1709.

Feldman, A. G. (1980). Superposition of motor programs - i. rhythmic forearm movements in man. Neuroscience, 5(1), 81-90.

Garfinkel, S. N., Seth, A. K., Barrett, A. B., Suzuki, K., \& Critchley, H. D. (2015). Knowing your own heart: distinguishing interoceptive accuracy from interoceptive awareness. Biological Psychology, 104, 65-74.

Goldenberg, M. S., Yack, H. J., Cerny, F. J., \& Burton, H. W. (1991). Acoustic myography as an indicator of force during sustained contractions of a small hand muscle. Journal of Applied Physiology, 70(1), 87-91.

Gu, Y., Yu, C., Li, Z., Li, W., Xu, S., Wei, X., \& Shi, Y. (2019). Accurate and low-latency sensing of touch contact on any surface with fingerworn IMU sensor (pp. 1059-1070). Proceedings of the 32nd Annual ACM Symposium on User Interface Software and Technology.

Gueorguiev, D., Bochereau, S., Mouraux, A., Hayward, V., \& Thonnard, J. L. (2016). Touch uses frictional cues to discriminate flat materials. Scientific Reports, 6, 25553.

Guigon, E., Chafik, O., Jarrasse, N., \& Roby-Brami, A. (2019). Experimental and theoretical study of velocity fluctuations during slow movements in humans. Journal of Neurophysiology, 121(2), 715-727.

Hipp, J., Arabzadeh, E., Zorzin, E., Conradt, J., Kayser, C., Diamond, M. E., \& Konig, P. (2006). Texture signals in whisker vibrations. Journal of Neurophysiology, 95(3), 1792-1799.

Hodges, P. W. (2019). Consensus for experimental design in electromyography (CEDE) project. Journal of Electromyography and Kinesiology In press.

Husserl, E. (1989). The constitution of psychic reality through the body (pp. 151-169). Ideas pertaining to a pure phenomenology and to a phenomenological philosophy, Springer.

Johansson, R. S., \& Westling, G. (1984). Roles of glabrous skin receptors and sensorimotor memory in automatic control of precision grip when lifting rougher or more slippery objects. Experimental Brain Research, 56, 550-564.

Jousmäki, V., \& Hari, R. (1998). Parchment-skin illusion: sound-biased touch. Current Biology, 8(6), 190-191.

Kilteni, K., \& Ehrsson, H. H. (2017). Body ownership determines the attenuation of self-generated tactile sensations. Proceedings of the National Academy of Sciences, 114(31), 8426-8431.

Kirkpatrick, S., Duncan, D. D., \& Fang, L. (2004). Low-frequency surface wave propagation and the viscoelastic behavior of porcine skin. Journal of Biomedical Optics, 9(6), 1311-1320.

Klöcker, A., Wiertlewski, M., Théate, V., Hayward, V., \& Thonnard, J.L. (2013). Physical factors influencing pleasant touch during tactile exploration. PloS One, 8(11), e79085.

Laput G., Xiao R. \& Harrison, C. (2016) Viband: High-fidelity bioacoustic sensing using commodity smartwatch accelerometers. In Proceedings of the 29th Annual Symposium on User Interface Software and Technology, pp. 321-333.

Latash, M. L., Scholz, J. P., \& Schöner, G. (2007). Toward a new theory of motor synergies. Motor Control, 11(3), 276-308.

Manfredi, L. R., Baker, A. T., Elias, D. O., Dammann III, J. F., Zielinski, M. C., Polashock, V. S., \& Bensmaia, S. J. (2012). The effect of surface wave propagation on neural responses to vibration in primate glabrous skin. PLoS One, 7(2), e31203.

Mannini, A., Intille, S. S., Rosenberger, M., Sabatini, A. M., \& Haskell, W. (2013). Activity recognition using a single accelerometer placed at the wrist or ankle. Medicine and Science in Sports and Exercise, 45(11), 2193.

McGlone, F., Wessberg, J., \& Olausson, H. (2014). Discriminative and affective touch: sensing and feeling. Neuron, 82(4), 737-755.

Merleau-Ponty, M. (1962). Phenomenology of perception. Routledge. 
Morris, J. R. W. (1973). Accelerometry - a technique for the measurement of human body movements. Journal of Biomechanics, 6(6), 729-736.

Morrison, I., Löken, L. S., \& Olausson, H. (2010). The skin as a social organ. Experimental Brain Research, 204(3), 305-314.

Ritt, J. T., Andermann, M. L., \& Moore, C. I. (2008). Embodied information processing: vibrissa mechanics and texture features shape micromotions in actively sensing rats. Neuron, 57(4), 599-613.

Schütz-Bosbach, S., Musil, J. J., \& Haggard, P. (2009). Touchant-touché: The role of self-touch in the representation of body structure. Consciousness and Cognition, 18(1), 2-11.

Schwarz, C. (2016). The slip hypothesis: tactile perception and its neuronal bases. Trends in Neurosciences, 39(7), 449-462.

Shao, Y., Hayward, V., \& Visell, Y. (2016). Spatial patterns of cutaneous vibration during whole-hand haptic interactions. Proceedings of the National Academy of Sciences, 113(15), 4188-4193.

Shao, Y., Hayward, V., \& Visell, Y. (2020). Compression of dynamic tactile information in the human hand. Science Advances, 6, eaaz1 158

Shergill, S. S., Bays, P. M., Frith, C. D., \& Wolpert, D. M. (2003). Two eyes for an eye: the neuroscience of force escalation. Science, 301(5630), 187-187.

Shi, Y., Zhang, H., Zhao, K., Cao, J., Sun, M., \& Nanayakkara, S. (2020). Ready, steady, touch! sensing physical contact with a fingermounted IMU. Proceedings of the ACM on Interactive, Mobile, Wearable and Ubiquitous Technologies, 4(2), 1-25.

Tanaka, Y., Horita, Y., Sano, A., \& Fujimoto, H. (2011). Tactile sensing utilizing human tactile perception. In IEEE World Haptics Conference (pp. 621-626). IEEE.

Tanaka, Y., Horita, Y., \& Sano, A. (2012). Finger-mounted skin vibration sensor for active touch. In P. Isokoski \& J. Springare (Eds.), Haptics: perception, devices, mobility, and communication (pp. 169-174). Berlin, Heidelberg: Springer.

Tronstad, C., Johnsen, G. K., Grimnes, S., \& Martinsen, Ø. G. (2010). A study on electrode gels for skin conductance measurements. Physiological Measurement, 31(10), 1395-1410.

Valderas, M. T., Bolea, J., Laguna, P., Vallverdù, M., \& Bailón, R. (2015). Human emotion recognition using heart rate variability analysis with spectral bands based on respiration. In 2015 37th Annual International Conference of the IEEE Engineering in Medicine and Biology Society (EMBC) (pp. 6134-6137). IEEE.

van Dooren, M., de Vries, J. J. G., \& Janssen, J. H. (2012). Emotional sweating across the body: Comparing 16 different skin conductance measurement locations. Physiology \& Behavior, 106(2), 298-304.

van Stralen, H. E., van Zandvoort, M. J. E., Hoppenbrouwers, S. S., Vissers, L. M. G., Kappelle, L. J., \& Dijkerman, H. C. (2014). Affective touch modulates the rubber hand illusion. Cognition, 131(1), 147-158.

Verrillo, R. T., Bolanowski, S. J., \& McGlone, F. P. (2003). Intra-and interactive touch on the face. Somatosensory \& Motor Research, 20(1), 3-11.

Vexler, A., Polyansky, I., \& Gorodetsky, R. (1999). Evaluation of skin viscoelasticity and anisotropy by measurement of speed of shear wave propagation with viscoelasticity skin analyzer. Journal of Investigative Dermatology, 113(5), 732-739.

Waris, A., Niazi, I. K., Jamil, M., Englehart, K., Jensen, W., \& Kamavuako, E. N. (2018). Multiday evaluation of techniques for EMG-based classification of hand motions. IEEE Journal of Biomedical and Health Informatics, 23(4), 1526-1534.

Wierda, S. M., van Rijn, H., Taatgen, N. A., \& Martens, S. (2012). Pupil dilation deconvolution reveals the dynamics of attention at high temporal resolution. Proceedings of the National Academy of Sciences, 109(22), 8456-8460.

Wiertlewski, M., Hudin, C., \& Hayward, V. (2011). On the 1/f noise and non-integer harmonic decay of the interaction of a finger sliding on flat and sinusoidal surfaces. in Proceedings of World Haptics Conference, pp. 25-30.

Wiertlewski, M., Lozada, J., Pissaloux, E., \& Hayward, V. (2010). Causality inversion in the reproduction of roughness. In A. M. L. Kappers, J. B. F. van Erp, W. M. Bergmann-Tiest, \& F. C. T. van der Helm (Eds.), Haptics: generating and perceiving tangible sensations (pp. 17-24). Berlin, Heidelberg: Springer.

Publisher's note Springer Nature remains neutral with regard to jurisdictional claims in published maps and institutional affiliations. 